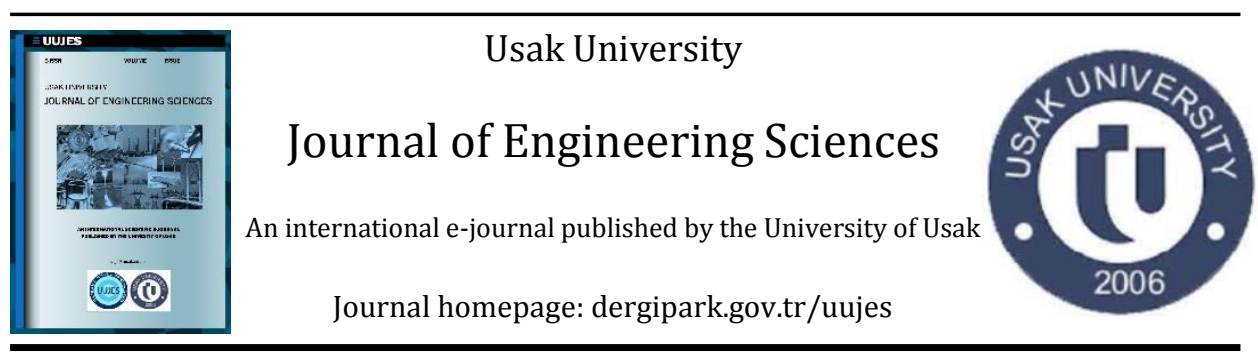

Research article

\title{
MONITORING OF BURN DAMAGE OCCURRENCE UNDER DIFFERENT GRINDING CONDITIONS USING ACOUSTIC EMISSION
}

\author{
Ísa Yesilyurt*, Onur Bakir \\ Usak University, Department of Mechanical Engineering, Usak,Turkey
}

Received: 12 Nov 2020

Revised: 6 Jan $2020 \quad$ Accepted: 15 Jan 2020

Online available: 28 Dec 2020

Handling Editor: Abdullah Yıldız

\begin{abstract}
This paper presents the effects of the operating conditions (i.e. cutting speed and the amount of infeed) in grinding on the occurrence of thermal damage to the workpiece. The paper commences with a brief background to the analysis methods. Firstly, the AE signals detected under different operating conditions are analysed in time and frequency domains. It has been found that an increase in cutting speed or feed rate increases the strength of AE activity which is clearly reflected by the statistical parameter. Moreover, the infeed rate rather than the speed of the cutting wheel is more influential on the occurrence of burn damage. When the infeed rate is increased, the workpiece exhibits symptoms of severe burn damage.
\end{abstract}

Keywords: Grinding, acoustic emission, burn damage; cutting speed; feed rate.

(C)2020 Usak University all rights reserved.

\section{Introduction}

Grinding, in its simplest form, is a chip removal process by scraping from the material surface with the help of sharp edges of abrasive particles and is the final manufacturing process where the desired surface quality and dimensional tolerances are achieved. During grinding, large cutting forces are generated which lead to elastic and plastic deformations in the workpiece [1]. In addition, an excessive heat is generated in the cutting zone and most of it flows into workpiece. As a result, the surface integrity of the workpiece may be damaged, and some failures such as burn and temper damage, rehardening damage and surface cracks may occur. There are many factors responsible for the increase in grinding temperature such as the type of workpiece material, the nature of abrasive employed in the grinding wheel, application of grinding fluid, grinding speeds

${ }^{*}$ Corresponding author: İsa Yeşilyurt

E-mail: isa.yesilyurt@usak.edu.tr (ORCID ID: 0000-0002-2329-748X)

DOI: $10.47137 /$ uujes.825105

(C)2020 Usak University all rights reserved. 
and feeds, and wheel dressing conditions [2]. Since grinding is a finish process and is usually the last stage of the manufacturing, any damage induced to the workpiece could be very costly.

A large number of research studies on tool condition monitoring have been reported in the literature for a variety of machining processes by measuring parameters such as force and torque, feed and spindle motor currents, acoustic emissions, vibration, cutting temperature, etc [3-8]. Of these parameters, acoustic emission (AE) has been widely used and recognised as one of the most suitable choices for grinding process monitoring. The use of acoustic emission is highly desirable due to its superior S/R ratio and sensitivity which lead to detection of different levels of $\mathrm{AE}$ activities occurring even at very low depths of cut. Another advantage is that the AE sensors generally operate within a frequency range of $50 \mathrm{kHz}-1000 \mathrm{kHz}$ well above the characteristic frequencies attributed to the machining or natural frequencies of structure and this minimises noise in the resulting AE signal [3-5].

Han et al. [6] studied characteristics of AE in precision grinding under different working conditions and justifications were made considering RMS and frequency spectrum of AE signal. Karpuschewski et al. [7] investigated the influence of different dressing parameters on the AE signal and wheel life estimation was achieved using power spectra of the enveloped AE signal. Plaza et al. [8] carried out an experimental study on acoustic emission (AE) features in abrasive grinding. The acoustic emission signals were analysed in both the time and frequency domains. It was found that the $\mathrm{AE}$ features in the frequency up to $200 \mathrm{kHz}$ could be an ideal data source for the online monitoring of surface creation in grinding processes. Aguiar et al. [9] investigated the efficiency of some statistical analysis methods in the detection of thermal damages in grinding process using AE signals.

In this study, the effects of the operating conditions (i.e. cutting speed and the amount of infeed) in grinding on the occurrence of thermal damage to the workpiece have been investigated using time and frequency domain analyses. The paper commences with a brief background to the analysis methods. Firstly, the AE signals detected under different operating conditions are analysed in time and frequency domains. It has been found that an increase in cutting speed or feed rate increases the strength of AE activity which is clearly reflected by the statistical parameter. Moreover, the feed rate rather than the cutting speed is more influential on the occurrence of burn damage. It has been found that the workpiece shows symptoms of temperature related damage severely when the infeed rate is increased.

\section{Materials and methods}

\subsection{Time domain analysis and statistical properties of a signal}

In time domain analysis, the time history of the signal itself can be used to determine the parameters of a vibration including peak value, signal energy, the time duration of events, statistical properties, the shaft orbit, and any modulation. Of these parameters, the statistical properties are often used as a basis for the fault detection [10]. Root mean square value $(R M S)$ of a signal $x(t)$ is used to indicate the power content of the signal. The $R M S$ is defined as:

$$
R M S=\sqrt{\int_{-\infty}^{\infty} x^{2}(t) d t}
$$


The root mean square is a single value that gives an idea of the power content of a signal. In some cases, the signal amplitude fluctuates rapidly with a small duration, and therefore the RMS value cannot truly reflect this spikiness. Crest factor and Kurtosis are used to indicate the spikiness of a signal and are defined respectively as:

$$
\begin{gathered}
F_{c}=\frac{P_{p}}{r m s} \\
K r=\frac{\int_{-\infty}^{\infty}[x(t)-\bar{x}]^{4} d t}{r m s^{4}}
\end{gathered}
$$

where $P_{p}$ and $\bar{x}$ denote peak-to-peak value (which is the difference between the maximum and minimum values of the signal) and mean value of the signal, being themselves defined as follow:

$$
\begin{gathered}
P_{p}=\max (x)-\min (x) \\
\bar{x}=\frac{1}{T} \int_{0}^{T} x(t) d t
\end{gathered}
$$

\subsection{Frequency domain analysis}

In frequency domain analysis, the amplitude of vibration response is represented against frequency. The vibration response of a machine is governed by factors which include its components, assembly, mounting, and operation. For these reasons, the vibration characteristics of any machine are unique to that particular machine, and provided that the excitation forces are constant, the measured vibration level will remain unchanged. It is for this reason that vibration spectra are often referred to as machine signatures. If the machine, however, starts to develop faults, its vibration level and consequently the shape of the frequency spectrum will change. By comparing the frequency spectrum of a machine in damaged condition with the reference frequency spectrum corresponding to the same machine in good condition, deterioration can be detected. The Fourier transform of a signal $x(t)$ is expressed as follows:

$$
X(f)=\int_{-\infty}^{\infty} x(t) e^{-j 2 \pi f t} d t
$$

where $f$ is the frequency variable. The spectral density of the signal per unit frequency at a particular frequency $f$ is $|X(f)|^{2}$, and total signal energy in the frequency domain can be calculated by summing up the spectral density function over all frequencies. According to Parseval's theorem, the total energy calculated in both the time and frequency domains is equal. That is:

$$
\int_{-\infty}^{\infty}|x(t)|^{2} d t=\int_{-\infty}^{\infty}|X(f)|^{2} d f
$$




\section{Results and discussions}

\subsection{Experimental Setup and Definitions of Process Parameters}

All the experiments were carried out on a plunge type CNC cylindrical grinding machine shown in Fig. 1. The workpiece used in grinding was a cam roller used for internal combustion engines. It was made of DIN $100 \mathrm{Cr} 6$ steel, case hardened to a depth of $0.15 \mathrm{~mm}$ at $60 \mathrm{HRC}$ and had an outer diameter of $32 \mathrm{~mm}$. A vitrified-bond aluminium oxide grinding wheel (whose diameter and thickness are $85 \mathrm{~mm}$ and $20 \mathrm{~mm}$ respectively) was used together with a $4 \%$ water soluble coolant.



Fig. 1 The roller guided and fixed on the work head of a cylindrical grinding machine

The condition of the grinding process was monitored for three different situations: normal grinding, grinding with higher cutting speed, and larger infeed. During the experiments, the speed of the workpiece was set to $500 \mathrm{rpm}$ for all cutting conditions. For the normal grinding test, the circumferential speed of the grinding wheel and the amount of infeed were selected as $57 \mathrm{~m} / \mathrm{sec}$ and $0.16 \mathrm{~mm} / \mathrm{sec}$ respectively. When a higher cutting speed is considered, the peripheral speed of grinding wheel was increased to $62 \mathrm{~m} / \mathrm{sec}$ without changing the feed rate. For the larger feed rate case, the feed rate was raised to $0.3 \mathrm{~mm} / \mathrm{sec}$ without altering the cutting speed.

An acoustic emission sensor, Kistler type 8152C, was used to capture the resulting signals. During the experiments, the obtained AE signals were sampled at $2 \mathrm{MHz}$ and captured for a complete grinding process which all last $7.5 \mathrm{sec}$, including idle runs before and after the grinding period. A built in anti aliasing filter in data acquisition board (centred at $85 \%$ of the sampling frequency) was used to avoid aliasing effect. To avoid machine related low frequency activities and high frequency noise, the AE signals were low-pass filtered on computer by a $10^{\text {th }}$ order elliptic filter whose cut-off frequencies are set at $3 \mathrm{kHz}$ and $800 \mathrm{kHz}$. 


\subsection{Time and Frequency Domain analyses of AE signals in Grinding}

Fig. 2 shows the AE signals and their corresponding spectra for three cases. It can be seen from the time variations for normal grinding that the amplitude of $\mathrm{AE}$ signal exhibits a systematic increase and reaches its maximum at the early stage of cutting period. This can be attributable to the fact that the depth of cut builds up after contact is made between the grinding wheel and the workpiece. This hence results in a gradual increase in undeformed chip thickness (and consequently cutting force) until the specified depth of cut is reached which leads to the larger deformation and raises the strength of AE. The amplitude of the AE signal decreases slightly to a certain value after reaching its maximum and then remains nearly constant until the end of the roughing period. In plunge type cylindrical grinding, the real depth of cut approaches the value of the set depth of cut after a few revolutions and remains constant unless feed and speed are kept unchanged. This consequently results in a constant undeformed chip thickness and yields a constant strength in AE activity. After roughing is finished, the amplitude of AE signal is reduced gradually until the end of the spark-out period. During roughing in plunge cylindrical grinding, a spiral shape rather than a round shape is obtained on the workpiece due to continuous radial infeed. A more nearly round part, desired size and surface quality are all obtained throughout the spark-out during which the wheel continues the grinding process on the workpiece without making any progress in radial direction. In the course of spark out, the undeformed chip thickness is diminished continuously reducing the strength of $\mathrm{AE}$ activity.

When the larger cutting speed is taken into account, the resulting time trace of AE signal exhibits very similar characteristics to those of the normal cutting with the exceptions that it gives larger RMS,crest, and kurtosis values as given in Table 1 (for the calculations of statistical values, only roughing and spark-out periods are taken into account). This is because the number of grinding particles in contact with workpiece rises with increasing wheel's peripheral speed which is equivalent to increase the number of acoustic emission sources and which causes a further increase in the strength of AE signal. Four tests were conducted to examine the effects of high grinding speed on thermal damage occurrence, and found that the application of a higher speed did not cause any thermal damage to the workpieces.

Increasing the amount of infeed from $0.16 \mathrm{~mm} / \mathrm{sec}$ to $0.30 \mathrm{~mm} / \mathrm{sec}$ reduces the roughing time, but raises the amplitude of AE especially at the early stage of roughing period. The main reason for this is that the undeformed chip thickness increases with the increase of the feed and therefore greater cutting forces occur. This leads to a significant increase not only in the number of active grains in contact with the workpiece, but in the strength of $\mathrm{AE}$ activities which cause further increases in the values of statistical parameters. Five tests were conducted to examine the effects of a larger infeed on thermal damage occurrence and it was found, unlike the higher cutting speed, that four out of five workpieces showed severe burn damage, whereas one exhibited mild burn damage.

Spectra of AE signals for all grinding conditions are illustrated up to $500 \mathrm{kHz}$ in Fig. 2. Frequency spectrum of $\mathrm{AE}$ signal for normal grinding exhibits three largest quasiperiodic peaks located at $25.68 \mathrm{kHz}, 86.3 \mathrm{kHz}$ and $146.4 \mathrm{kHz}$. When the spectrum is zoomed around the first largest peak, there exists a family of sidebands located at $206.67 \mathrm{~Hz}$ intervals from each other. It can hence be concluded that the resulting $\mathrm{AE}$ signal is modulated by the rotation of the grinding wheel with a frequency of $206.67 \mathrm{~Hz}$ (i.e. $12400 \mathrm{rev} / \mathrm{min}$ ). A very fine details about the frequency component around $25.68 \mathrm{kHz}$ are illustrated in Fig. 3. It can be seen that there is another family of sidebands positioned at $8.33 \mathrm{~Hz}$ intervals around the all of those $206.67 \mathrm{~Hz}$ peaks. Because the rotational speed 
of workpiece is $501 \mathrm{rev} / \mathrm{min}$ (i.e. $8.35 \mathrm{~Hz}$ ), the signal due to the rotation of wheel is further modulated by the speed of workpiece.

When the peripheral speed of grinding wheel is increased to $62 \mathrm{~m} / \mathrm{sec}$, the frequency positions of the quasi-periodic peaks are not changed, but their magnitudes are reduced notably. However, the increase in speed is influential on the characteristics of both sideband families. Sidebands occurring due to the rotational speed of the wheel are spaced with nearly $225 \mathrm{~Hz}$ intervals and magnitudes of sidebands spaced with $8.33 \mathrm{~Hz}$ intervals are raised. Increasing the amount of infeed to $0.3 \mathrm{~mm} / \mathrm{sec}$ is not influential on the frequency positions of the quasi-periodic peaks again, but their magnitudes are further diminished. Since both the wheel and workpiece speeds are kept the same as those for normal cutting, there is no change in the positions of sideband activities, but the magnitudes of the sidebands spaced at $8.33 \mathrm{~Hz}$ intervals become even stronger.
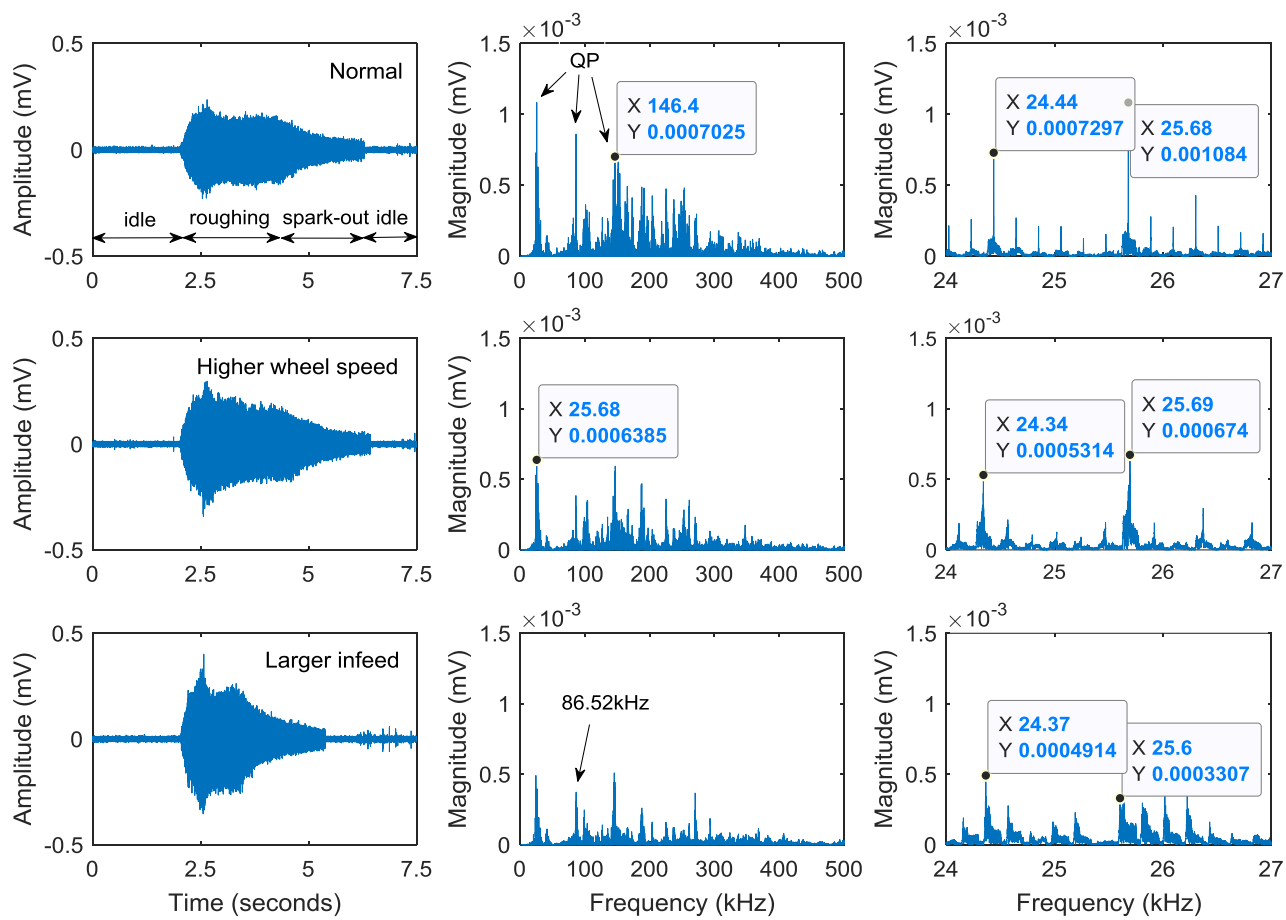

Fig. 2 Time and frequency domain representations of AE signals for normal grinding (first row), higher cutting speed (second row) and larger infeed (third row) 




Fig. 3 A very close look at the spectrum of the normal grinding AE activity around $25.6 \mathrm{kHz}$

Table 1 Statistical values of AE activities for different grinding conditions

\begin{tabular}{lccc}
\hline Grinding condition & RMS & Crest & Kurtosis \\
\hline Normal & 0.031 & 15.00 & 4.56 \\
Higher wheel speed & 0.039 & 16.38 & 4.84 \\
Larger infeed & 0.043 & 17.58 & 5.69 \\
\hline
\end{tabular}

\section{Conclusions}

In this study, condition of grinding process has been monitored using AE under different operating conditions and the following conclusions can be drawn.

The strength of $\mathrm{AE}$ increases when a higher wheel speed or greater amount of feed rate is applied in grinding. This is because the higher the wheel speed, the more abrasive particles are in contact with the workpiece, increasing the number of AE sources. Similarly, as the feed rate increases, the instantaneous chip thickness becomes larger, resulting in a bigger cutting force and greater AE amplitude. Also, any increase in wheel speed or feed rate leads to an increase in RMS, crest and Kurtosis values of the $\mathrm{AE}$ activity.

It has been found that the feed rate rather than the speed of the wheel is highly influential in the occurrence of thermal damage. When a higher wheel speed is applied, none of the workpieces exhibits any burn damage. When a larger amount of infeed is applied, four of the five workpieces have shown symptoms of severe burn damage, while one has exhibited mild burn damage.

The spectra of AE signals for all grinding conditions exhibit three main quasi-periodic peaks located at $25.68 \mathrm{kHz}, 86.3 \mathrm{kHz}$ and $146.4 \mathrm{kHz}$, but the positions of these frequencies are neither speed nor feed dependent. A detailed examination of spectra reveals that there exists a family of sidebands due to the rotation of the wheel whose peaks are located at $206.67 \mathrm{~Hz}$ intervals from each other. Also, due to the rotation of the workpiece, there exists another family of sideband around those $206.67 \mathrm{~Hz}$ peaks located at $8.33 \mathrm{~Hz}$ intervals. Although the amplitudes of those quasi-periodic frequency peaks are reduced notably when the cutting speed or feed rate is increased, the peaks of sideband family located at $8.33 \mathrm{~Hz}$ intervals are strengthened significantly.

\section{Acknowledgments}

The authors wish to express their appreciation to Ortadogu Rulman Sanayii (ORS) in Ankara-Turkey, for their valuable assistance and support given to this work. 


\section{References}

1. Grote KH and Antonsson EK. Springer handbook of mechanical engineering. Springer-Verlag Berlin: Heidelberg; 2009.

2. Brian RW. Principles of modern grinding technology. $2^{\text {nd }}$ edition. Elsevier: 2014.

3. Dimla A and Dimla S. Sensor signals for tool-wear monitoring in metal cutting operations-a review of methods. Int J Mach Tools \& Manuf, 2000;40:1073-1098.

4. Lauro CH, Brandao LC, Baldo D, at al. Monitoring and processing signal applied in machining processes-A review. Measurement,2014;58:73-86.

5. Babel RJP. Acoustic emission spikes at workpiece edges in grinding: origin and applications. MsC Thesis, McMaster University, Canada, 2011.

6. Han $\mathrm{X}$ and $\mathrm{Wu} \mathrm{T}$. Analysis of acoustic emission in precision and high-efficiency grinding technology. Int J Adv Manuf Technology, 2013;67:1997-2006.

7. Karpuschewski B and Wehmeier M. Grinding monitoring system based on power and acoustic emission sensors. Ann CIRP 2000;49(1):235-240.

8. Plaza EG, Chen X, Ouarab LA, at al. Abrasive feature related acoustic emission in grinding. In: Proceedings of the 25th international conference on Automation \& Computing; 2019 Sep 5-7; Lancaster UK: p. 1-6.

9. Aguiar PR, Serni PJA, Bianchi EC, at al. In-process grinding monitoring by acoustic emission. In: IEEE International conference on acoustics, speech, and signal processing., 2004 May 17-21; Montreal, Quebec, Canada, p. 405-408.

10. Yesilyurt I. Gearbox fault detection and severity assessment using vibration analysis, PhD. Thesis, University of Manchester, UK, 1997. 